\title{
The Impact of Sexual Orientation on College Student Victimization: An Examination of Sexual Minority and Non-Sexual Minority Student Populations
}

\author{
Mark Beaulieu1, Creaig Dunton¹, LaVerne McQuiller Williams², Judy L. Porter ${ }^{2}$ \\ ${ }^{1}$ State University of New York College at Plattsburgh, Plattsburgh, USA \\ ${ }^{2}$ Rochester Institute of Technology, Henrietta, USA \\ Email: *mbeau002@plattsburgh.edu
}

How to cite this paper: Beaulieu, M., Dunton, C., Williams, L. M., \& Porter, J. L. (2017). The Impact of Sexual Orientation on College Student Victimization: An Examination of Sexual Minority and Non-Sexual Minority Student Populations. Psychology, 8, 1728-1747. https://doi.org/10.4236/psych.2017.811114

Received: July 31, 2017

Accepted: September 5, 2017

Published: September 8, 2017

Copyright $\odot 2017$ by authors and Scientific Research Publishing Inc. This work is licensed under the Creative Commons Attribution International License (CC BY 4.0).

http://creativecommons.org/licenses/by/4.0/

(c) (i) Open Access

\begin{abstract}
Our research examines the possible association of sexual orientation and self-reported sexual harassment, sexual assault, intimate partner psychological abuse, and intimate partner physical abuse of college students from a northeastern university in the United States. Understanding the prevalence of these behaviors within this age group is important, as developing appropriate college policy to reduce these actions at this time may be beneficial for future prevention. This study also allows for one of the few true comparisons between those students who identified as sexual minority and non-sexual minority college students ${ }^{1}$. A total of 1881 college students were surveyed. Classes were randomly selected and surveys were administered to students in those classes. Gender specific models were also analyzed to examine if the relationship between sexual orientation and victimization differs for males and females. The results from the analyses support the prediction that sexual minority students were more likely to report all four types of victimization than non-sexual minority students. Sexual minority students were about four times more likely to report sexual assault, two times more likely to report sexual harassment and physical abuse, and about 1.5 times more likely to report psychological abuse. Sexual minority males were more likely to report sexual assault (nine times), sexual harassment (3.5 times), and physical abuse (twice as likely). Sexual minority females were about twice as likely to report sexual assault, physical abuse, and psychological abuse.
\end{abstract}

\section{Keywords}

IPV, Sexual Minority, Sexual Orientation, Gender, Women, Sexual Assault,

${ }^{1}$ The survey provided the following choices under the question about sexual orientation: Gay, Lesbian, Bi-sexual, and other. 
Sexual Harassment, Physical Abuse, Psychological Abuse

\section{Introduction}

\subsection{Intimate Partner Violence}

Intimate partner violence (IPV) is a pervasive public health concern that has received significant attention over the past three decades. IPV is an umbrella term for psychological, physical, and sexual abuse experienced between couples of any status that are in a relationship with one another (World Health Organization 2012). Research suggests that young adults between ages 18 - 24 experience the highest rates of IPV, and that college students are at an increased risk for some forms of IPV (e.g., sexual assault) when compared with same-age non-college peers (Fisher, Cullen, \& Turner, 2000; Justice, 2000).

Despite a large body of literature documenting rates of IPV among college students in general, previous research has focused largely on experiences of heterosexual college students, often ignoring those of sexual minorities. The importance of examining sexual orientation in the context of IPV cannot be understated as research has suggested that sexual minority college students are significantly more likely to report dating violence and sexual assault than non-sexual minority students (Edwards et al., 2015). Thus, the current study examines whether or not sexual minority college students are more likely to be victims of sexual harassment, sexual assault, and intimate partner physical and psychological abuse than non-sexual minority students. In addition, to provide a clearer understanding of IPV among sexual minorities, the study also examined whether there were gender differences for males and females.

Studies focusing on IPV among heterosexual college samples suggest that almost one third of college students have reported some experience with physical abuse within a romantic relationship (Orcutt, Garcia, \& Pickett, 2005; Perry \& Fromuth, 2005). More common is the presence of psychological abuse, with some studies reporting up to $80 \%$ of college aged couples experiencing this type of victimization (Avant, Swopes, Davis, \& Elhai, 2011; Cercone, Beach, \& Arias, 2005; Harned, 2001; Hines \& Saudino, 2003). Although male and female college students report similar rates of physical and psychological abuse by a partner, female college students report sexual assault at higher rates than male college students. To this end, researchers have found 1-year incidence rates of sexual assault for college women ranging from $17 \%$ to $33 \%$ (Smith, White, \& Holland, 2003). Conversely, $8.2 \%$ of college men reported sexual assault (Banyard et al., 2007).

\subsection{Sexual Minorities and Victimization}

Another of the most frequently cited victimization concerns on college campuses is sexual assault among both sexual minorities and sexual non-minorities. While 
women are more often the victims of sexual assault than men, both gay males and lesbians are significantly over represented as victims (Duncan, 1990). A meta-analysis of articles regarding gay, lesbian, and bisexual victims of sexual assault showed elevated rates of victimization, with gay males having $11.8 \%$ to $54 \%$ victimization rates, compared to two to three percent among heterosexual males. Lesbians were victimized at rates of $15.6 \%$ to $85 \%$, compared to $11 \%$ to $17 \%$ of heterosexual women (Rothman, Exner, \& Baughman, 2011; Todahl, Linville, Wheeler, \& Gau, 2009; Wilson \& Spatz Widom, 2010). However, critiques of this research have argued that most of these studies did not discern between same sex victimization and heterosexual victimization, thus making the findings unclear if the perpetrator was the same sex as the victim or not (Waldner-Haugrud \& Vaden Gratch, 1997). Also, it should be noted that the bulk of these studies were not direct comparisons between gay, lesbian and bisexual victims to heterosexual victims, and the theoretical reasons for these differences have largely been ignored (Rothman et al., 2011). This research does, however, compare the two groups to one another to determine the role played by sexual orientation in victimization.

Research on psychological abuse among sexual minorities in non-college populations also reveals high rates of abuse. For example, studies of lesbians have found that verbal and psychological abuse was prevalent, ranging from 73 to $90 \%$ of women studied. This was consistent with gay males as well, with as many as $95 \%$ reporting at least one incident of psychological abuse, indicating a higher frequency of victimization (Bartholomew et al., 2008; Burke \& Follingstad, 1999; Lockhart et al., 1994). However, it should be noted that the definition of psychological abuse is less universally defined than physical abuse, and thus what constitutes abuse may differ from study to study (Follingstad \& DeHart, 2000).

Of additional concern are directly aimed forms of harassment, sexual and otherwise, toward sexual minority students based upon their sexual orientation. An early study of harassment of gay and lesbian students in colleges found that over $77 \%$ experienced verbal harassment regarding their sexual orientation at some point, with $49 \%$ reporting more than one incident of verbal harassment while a student. However, this study surveyed only gay and lesbian students (D’Augelli, 1992). Two decades later, harassment based upon sexual orientation has continued to be a problem amongst sexual minority students, with $36 \%$ reporting being harassed in the previous year for their orientation (Rankin, 2003).

\subsection{College Life and Risk Factors for Victimization}

A number of control variables-living alone, living on campus, race, and participation in sports-are also important to acknowledge when studying victimization amongst college age populations. Previous research suggests that living arrangements may be associated with victimization risk, whereby college students that live independently and off-campus may be more vulnerable than those living with others and on-campus (Anderson \& Leigh, 2010; Lehrer, Lehrer, Lehrer, \& 
Oyarzun, 2007). Some research also suggests that race may be associated with the risk of victimization, although studies are inconclusive. For example, some research has found higher rates of interpersonal violence among African Americans when compared to Whites (Caetano, Schafer, \& Cunradi, 2001; Makepeace, 1987; Rouse, 1988) while another study found just the opposite (Lane \& Gwartney-Gibbs, 1985).

Although not studied extensively, it has also been suggested that participation in sports may protect females from victimization in that it develops strength and self-esteem (Fasting, Brackenridge, \& Sungot-Borgen, 2003). An often held assumption is that fraternity and sports membership are significant correlates for perpetrating sexually aggressive acts, largely due to hyper masculine beliefs, secrecy, and peer pressure (Boeringer, 1996; Martin \& Hummer, 1989). Recent research has found, however, that fraternity and sports membership has more of an indirect effect based upon those groups' emphasis on maintaining secrecy and peer pressure as related to sexual behaviors (Franklin, Bouffard, \& Pratt, 2012). Alcohol consumption, closely associated with fraternity and sorority participation, has also been found to be a significant predictor of sexual victimization (Monks, Tomaka, Palacios, \& Thompson, 2010).

Research also suggests that international students also may be targeted more frequently due to language barriers and the fact they are not as assimilated into American culture when compared to non-international students. This sometimes drastic change in cultural roles and expectations, coupled with a general fear of strangers, can lead to feelings of helplessness and an increased vulnerability to victimization (Coston, 2004).

\subsection{Current Study}

This study adds to the literature by examining how both sexual orientation and negative attitudes towards orientation affects various victimizations (physical and psychological abuse, sexual harassment and sexual assault) on a college campus. These are the institutions one would expect to be most tolerant and progressive regarding social issues of this type, but that often is not the case (M. J. Brown \& Groscup, 2009; Cotten-Huston \& Waite, 2000; Rankin, 2003). We also examine the effect of sexual orientation on victimization by gender.

The primary goal of this research is to determine the nature of victimization amongst college students, and how their gender and sexual orientation may be a factor in their victimization. Understanding the experiences on a college campus will provide insight as to what importance having clear and enforceable policies at the administrative level may have. As previously mentioned, many of the previous studies have used vague and changing definitions for what constitutes abuse and harassment, thus making comparisons between studies difficult. Considering previous research has not included both sexual minority and non-sexual minority populations together, this research makes these comparisons possible since both populations are studied using the same instruments. 
Based upon the extant literature, it is hypothesized that sexual minority students will be more likely to report victimization experiences in all of the categories under study. It is also expected that females will experience the highest levels of IPV victimization overall, while men will report the lowest levels of victimization regardless of type of relationship. With regard to sexual assault alone, it is expected that non-sexual minority females will experience higher rates of sexual victimization compared to sexual minority females, while sexual minority males are hypothesized to experience higher rates of sexual victimization compared to non-sexual minority males. It is also expected that same sex couples will report the highest rates of sexual harassment, with non-sexual minority females reporting more incidents than males.

\section{Methods}

\subsection{Data Collection}

The cross-sectional data for this study were collected from students at the Rochester Institute of Technology (RIT) (http://www.rit.edu/), a northeastern university in the United States. Forty classes were randomly selected by the researchers. After receiving approval from the Institutional Review Board (IRB) at RIT, surveys were distributed within randomly selected classes to all students. Participants were informed that the survey was voluntary and were told that if they had previously filled out a survey they should not fill out the survey again. A total of 1941 students filled out the surveys for response rates of $97.2 \%$ in year $1(\mathrm{n}=954)$ and $98.3 \%(\mathrm{n}=987)$ in year 2 . After removing cases missing data on any of the dependent and independent variables, the final sample size for this study was 1881 .

There were some other representativeness issues that should be addressed for these surveys. First, women were slightly overrepresented in the sample. Women were $33.4 \%$ of the student population. In the sample used for this study, women accounted for about $40 \%(39.2 \%)$ of the participants. The different colleges at the university were not represented in the sample as they were within the student population. In particular, the College of Liberal Arts was oversampled within the survey and this college has a higher percentage of women than the other colleges at the university. The reason for the oversampling was that the College of Liberal Arts classes were selected since all students are required to take these courses. It was the most efficient way to guarantee the inclusion of students from every college.

\subsection{Participants}

The sample consisted of 1881 respondents, including 1143 men (60.8\%) and 738 women $(39.2 \%)$ (see Table 1$)$. Slightly over $6 \%(n=115)$ identified as a sexual minority based upon our definition. The majority of the participants $(74.8 \%)$ identified themselves as White. There were 107 (5.7\%) international students. More than half of the respondents lived on campus $(54.7 \% ; n=1029)$ and about 
Table 1. Frequency distributions.

\begin{tabular}{|c|c|c|c|c|c|c|c|}
\hline \multirow[b]{2}{*}{ Variable } & \multirow[b]{2}{*}{ Value } & \multicolumn{2}{|c|}{ All Cases $(\mathrm{N}=1881)$} & \multicolumn{2}{|c|}{ Males $(\mathrm{N}=1143)$} & \multicolumn{2}{|c|}{ Females $(\mathrm{N}=738)$} \\
\hline & & Frequency & Percent & Frequency & Percent & Frequency & Percent \\
\hline \multirow{2}{*}{ Sexual Assault } & No & 1678 & $89.21 \%$ & 1062 & $92.91 \%$ & 616 & $83.47 \%$ \\
\hline & Yes & 203 & $10.79 \%$ & 81 & $7.09 \%$ & 122 & $16.53 \%$ \\
\hline \multirow{2}{*}{ Sexual Harassment } & No & 951 & $50.56 \%$ & 737 & $64.48 \%$ & 214 & $29.00 \%$ \\
\hline & Yes & 930 & $49.44 \%$ & 406 & $35.52 \%$ & 524 & $71.00 \%$ \\
\hline \multirow{2}{*}{ Physical Abuse } & No & 1466 & $77.94 \%$ & 887 & $77.60 \%$ & 579 & $78.46 \%$ \\
\hline & Yes & 415 & $22.06 \%$ & 256 & $22.40 \%$ & 159 & $21.54 \%$ \\
\hline \multirow{2}{*}{ Psychological Abuse } & No & 1135 & $60.34 \%$ & 718 & $62.82 \%$ & 417 & $56.50 \%$ \\
\hline & Yes & 746 & $39.66 \%$ & 425 & $37.18 \%$ & 321 & $43.50 \%$ \\
\hline \multirow{2}{*}{ Sexual Orientation } & Heterosexual & 1766 & $93.89 \%$ & 1095 & $95.80 \%$ & 671 & $90.92 \%$ \\
\hline & LGBT & 115 & $6.11 \%$ & 48 & $4.20 \%$ & 67 & $9.08 \%$ \\
\hline \multirow{2}{*}{ Gender } & Male & 1143 & $60.77 \%$ & - & - & - & - \\
\hline & Female & 738 & $39.23 \%$ & - & - & - & - \\
\hline \multirow{2}{*}{ Belongs to a Fraternity/Sorority } & No & 1659 & $88.20 \%$ & 1007 & $88.10 \%$ & 652 & $88.35 \%$ \\
\hline & Yes & 222 & $11.80 \%$ & 136 & $11.90 \%$ & 86 & $11.65 \%$ \\
\hline \multirow{2}{*}{$\begin{array}{c}\text { On a Sports Team } \\
\text { (Intercollegiate/Campus) }\end{array}$} & No & 1567 & $83.31 \%$ & 924 & $80.84 \%$ & 643 & $87.13 \%$ \\
\hline & Yes & 314 & $16.69 \%$ & 219 & $19.16 \%$ & 95 & $12.87 \%$ \\
\hline \multirow{2}{*}{ International Student } & No & 1774 & $94.31 \%$ & 1083 & $94.75 \%$ & 691 & $93.63 \%$ \\
\hline & Yes & 107 & $5.69 \%$ & 60 & $5.25 \%$ & 47 & $6.37 \%$ \\
\hline \multirow{2}{*}{ Lives on Campus } & No & 852 & $45.30 \%$ & 499 & $43.66 \%$ & 353 & $47.83 \%$ \\
\hline & Yes & 1029 & $54.70 \%$ & 644 & $56.34 \%$ & 385 & $52.17 \%$ \\
\hline \multirow{2}{*}{ Lives Alone } & No & 1686 & $89.63 \%$ & 1033 & $90.38 \%$ & 653 & $88.48 \%$ \\
\hline & Yes & 195 & $10.37 \%$ & 110 & $9.62 \%$ & 85 & $11.52 \%$ \\
\hline \multirow{2}{*}{ Race } & Non-white & 475 & $25.25 \%$ & 245 & $21.43 \%$ & 230 & $31.17 \%$ \\
\hline & White & 1406 & $74.75 \%$ & 898 & $78.57 \%$ & 508 & $68.83 \%$ \\
\hline
\end{tabular}

$10 \%(\mathrm{n}=195)$ lived alone. Only a small percentage of the sample, $16.7 \%(\mathrm{n}=$ $314)$, participated in intercollegiate or campus sports or belonged to a fraternity or sorority $(11.8 \% ; \mathrm{N}=222)$.

\subsection{Measures}

The dependent variables for analysis are dummy variables created from a variety of questions pertaining to various types of victimization. The first type of victimization is sexual harassment. A student that reported experiencing one or more of the following over the past year received a score of one on this variable: unwelcome remarks of a sexual nature from students, unwelcome remarks of a sexual nature from faculty, unwelcome touching from students, unwelcome touching from faculty/staff, repeated pressure for dates/sexual activity from students, and repeated pressure for dates/sexual activity from faculty/staff (Cronbach's Alpha = 0.58). 
The second form of victimization examined was sexual assault. Any student that reported verbal threats of sex against their will, sexual touching against their will, attempted sexual penetration (vaginal, oral, and/or anal) against their will, and/or sexual penetration (vaginal, oral, and/or anal) against their will were recorded as having been sexually assaulted (Cronbach's Alpha $=0.72$ ). Participants reported sexual victimization that occurred over the school year in the Sexual Experiences Survey (SES) (Koss, Gidycz, \& Wisniewski, 1987). The SES asks about a variety of sexually-related behaviors including verbal coercion, authority abuse, and acts legally defined as attempted rape and sexual assault. Thus, our measure is consistent with a past measure of sexual abuse.

To measure psychological and physical abuse among dating partners within the past school year, Straus et al.'s (1996) Revised Conflict Tactics Scale (CTS2) was used to measure intimate partner violence by "a partner" over the previous school year. Use of the term "partner" denotes intimate partner violence may exist among non-sexual minority and same-sex partners. The CTS2 is a commonly used measure of intimate partner violence that measures the frequency with which respondents had experienced psychological and physical abuse from their dating partners. Three items assessed psychological abuse: partner insulted or swore at you, partner put you down in front of friends and/or family, and partner threatened to hit/throw something at you (Cronbach's Alpha $=0.60$ ). Seven items assessed physical abuse: partner pushed, grabbed or shoved you, partner slapped you, partner kicked, bit, or shoved you, partner beat you up, partner hit you or tried to hit you with something, partner choked you, and partner threatened you with a gun/knife (Cronbach's Alpha $=0.75$ ). Subjects responded on a four-point scale (never, 1 - 2 times, 3 - 10 times, more than 10 times).

Sexual orientation was measured with the following question: "Which of the following best describes your sexual orientation?" Heterosexual was coded as 0 and gay, lesbian, bisexual, or other were coded as 1 . Gender was coded as a dummy variable where $1=$ women and $0=$ men. As discussed in the introduction, a variety of control variables were included due to prior research that has found a relationship between these variables and victimization. The control variables were all made into dummy variables and coded as follows: involvement in sports $(1=$ intercollegiate or campus; $0=$ none); international student $(1=$ yes; $0=$ no); live on campus ( $1=$ on campus; 0 = off campus or other $)$; live alone ( 1 = alone; 0 = roommate/housemate, or spouse/significant other, parents, and other $)$; and $\operatorname{race}(1=$ White; $0=$ non White $)$.

Table 2 presents the mean and standard deviations for the full sample and for subsamples based on gender. The correlation matrices are provided in Tables $3(\mathrm{a})-(\mathrm{c})$. A correlation matrix is provided for each subsample based on gender. The correlations between the various measures of reported abuse are significant, except for between sexual assault and physical abuse for men.

The victimization variables are all dummy variables that indicate the presence or absence of abuse. While a scale could have been used since there are three 
Table 2. Univariate statistics.

\begin{tabular}{|c|c|c|c|c|c|c|}
\hline \multirow[b]{2}{*}{ Variable } & \multicolumn{2}{|c|}{ All Cases $(\mathrm{N}=1881)$} & \multicolumn{2}{|c|}{ Males $(\mathrm{N}=1143)$} & \multicolumn{2}{|c|}{ Females $(\mathrm{N}=738)$} \\
\hline & Mean & $\begin{array}{l}\text { Standard } \\
\text { Deviation }\end{array}$ & Mean & $\begin{array}{l}\text { Standard } \\
\text { Deviation }\end{array}$ & Mean & $\begin{array}{l}\text { Standard } \\
\text { Deviation }\end{array}$ \\
\hline Sexual Assault & 0.108 & 0.310 & 0.071 & 0.257 & 0.165 & 0.372 \\
\hline Sexual Harassment & 0.494 & 0.500 & 0.355 & 0.479 & 0.710 & 0.454 \\
\hline Physical Abuse & 0.221 & 0.415 & 0.224 & 0.417 & 0.215 & 0.411 \\
\hline Psychological Abuse & 0.397 & 0.489 & 0.372 & 0.484 & 0.435 & 0.496 \\
\hline $\begin{array}{c}\text { Sexual orientation } \\
(\text { Heterosexual }=0 ; \mathrm{LGBT}=1)\end{array}$ & 0.061 & 0.240 & 0.042 & 0.201 & 0.091 & 0.287 \\
\hline $\begin{array}{c}\text { Gender }(\text { Male }=0 ; \\
\text { Female }=1)\end{array}$ & 0.392 & 0.488 & 0.000 & 0.000 & 1.000 & 0.000 \\
\hline $\begin{array}{c}\text { Belongs to a } \\
\text { Fraternity/Sorority } \\
(\text { No }=0 ; \text { Yes }=1)\end{array}$ & 0.118 & 0.323 & 0.119 & 0.324 & 0.117 & 0.321 \\
\hline $\begin{array}{c}\text { On a sports team } \\
\text { (Intercollegiate or Campus) } \\
(\mathrm{No}=0 ; \text { Yes }=1)\end{array}$ & 0.167 & 0.373 & 0.192 & 0.394 & 0.129 & 0.335 \\
\hline $\begin{array}{l}\text { International Student } \\
\quad(\text { No }=0 ; \text { Yes }=1)\end{array}$ & 0.057 & 0.232 & 0.052 & 0.223 & 0.064 & 0.244 \\
\hline $\begin{array}{l}\text { Lives on Campus } \\
(\text { No }=0 ; \text { Yes }=1)\end{array}$ & 0.547 & 0.498 & 0.563 & 0.496 & 0.522 & 0.500 \\
\hline Live Alone $($ No $=0 ;$ Yes $=1)$ & 0.104 & 0.305 & 0.096 & 0.295 & 0.115 & 0.319 \\
\hline $\begin{array}{l}\text { Race }(\text { Non-white }=0 ; \\
\quad \text { White }=1)\end{array}$ & 0.747 & 0.435 & 0.786 & 0.411 & 0.688 & 0.463 \\
\hline
\end{tabular}

psychological abuse measures and seven physical abuse measures, the main purpose of this study was to see if there was a difference in the reporting of abuse. Dummy variables allow for the testing of the presence/absence of each type of abuse. Additionally, the scales had low Cronbach's Alphas, particularly for psychological abuse, and skewed distributions. Chi-square statistics were calculated to test for differences in the prevalence of each of the types of victimization experienced. Binary logistic regression analyses were used to examine the effect of sexual orientation, gender, and other variables on the two types of victimization. Columns three and four examine the effects of the independent variables on psychological and physical abuse for men and women. Multicollinearity does not appear to be an issue for any of the models. The largest variance inflation factor (VIF) in any of the various models was 1.14. Data were analyzed using SPSS version 17 (SPSS Inc., 2008).

\section{Results}

Tables 4(a)-(d) provide the results for the binary logistic regressions for the various types of abuse. Sexual orientation has a significant effect on the likelihood of reporting sexual assault. This finding holds for the sample with both 
Table 3. (a) Correlations for all cases $(\mathrm{N}=1881)$. (b) Correlations for males $(\mathrm{N}=1143)$. (c) Correlations for females $(\mathrm{N}=738)$.

(a)

\begin{tabular}{|c|c|c|c|c|c|c|c|c|c|c|c|c|}
\hline 营 & $\begin{array}{l}\text { 苛 } \\
\text { 岁 } \\
\text { 褐 } \\
\text { 心 }\end{array}$ & 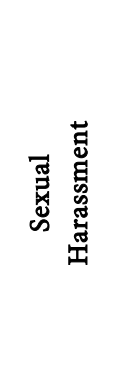 & 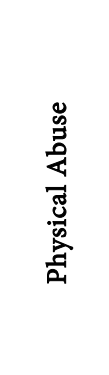 & 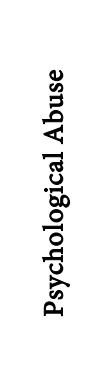 & 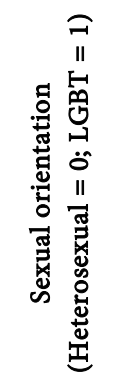 & 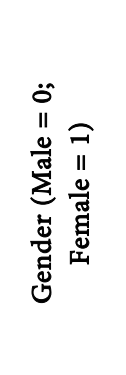 & 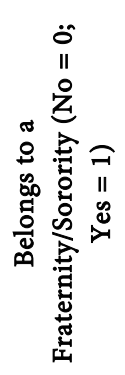 & 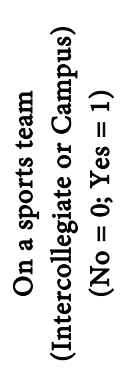 & 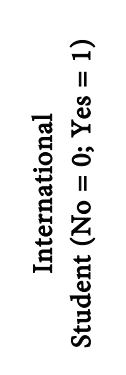 & 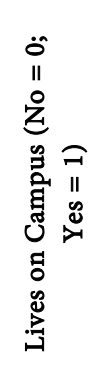 & 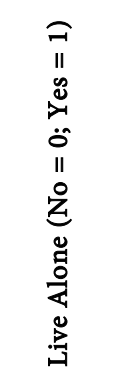 & 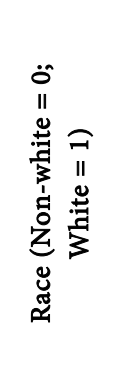 \\
\hline $\begin{array}{c}\text { Sexual } \\
\text { Assault }\end{array}$ & 1.000 & $0.245^{\star \star}$ & $0.162^{\star *}$ & $0.121^{\star *}$ & $0.183^{* *}$ & $0.149^{\star *}$ & $0.064^{\star *}$ & 0.028 & $0.048^{*}$ & $0.048^{\star}$ & $0.107^{\star \star}$ & $-0.090^{\star *}$ \\
\hline $\begin{array}{c}\text { Sexual } \\
\text { Harassment }\end{array}$ & $0.245^{\star *}$ & 1.000 & $0.112^{\star *}$ & $0.218^{* *}$ & $0.107^{\star *}$ & $0.347^{* *}$ & $0.077^{\star \star}$ & -0.004 & 0.042 & $0.067^{\star *}$ & 0.030 & $-0.076^{\star *}$ \\
\hline $\begin{array}{l}\text { Physical } \\
\text { Abuse }\end{array}$ & $0.162^{\star *}$ & $0.112^{\star *}$ & 1.000 & $0.384^{* *}$ & $0.078^{* *}$ & -0.010 & 0.044 & 0.016 & 0.035 & 0.000 & 0.029 & $-0.048^{\star}$ \\
\hline Psychological Abuse & $0.121^{\star *}$ & $0.218^{\star *}$ & $0.384^{* *}$ & 1.000 & $0.074^{\star *}$ & $0.063^{* *}$ & $0.054^{*}$ & -0.010 & 0.017 & $-0.055^{*}$ & 0.042 & -0.032 \\
\hline $\begin{array}{c}\text { Sexual } \\
\text { orientation } \\
(\text { Heterosexual }=0 ; \\
\text { LGBT }=1)\end{array}$ & $0.183^{\star *}$ & $0.107^{\star \star}$ & $0.078^{\star *}$ & $0.074^{\star *}$ & 1.000 & $0.099^{* *}$ & $0.051^{\star}$ & -0.043 & $0.110^{\star *}$ & -0.044 & $0.066^{\star *}$ & $-0.082^{\star *}$ \\
\hline $\begin{array}{c}\text { Gender } \\
(\text { Male }=0 ; \\
\text { Female }=1)\end{array}$ & $0.149^{\star *}$ & $0.347^{\star \star}$ & -0.010 & $0.063^{* *}$ & $0.099^{* *}$ & 1.000 & -0.004 & $-0.082^{\star *}$ & 0.024 & -0.041 & 0.030 & $-0.109^{\star *}$ \\
\hline $\begin{array}{c}\text { Belongs to a } \\
\text { Fraternity/Sorority } \\
\begin{array}{c}(\text { No }=0 ; \\
\text { Yes }=1)\end{array}\end{array}$ & $0.064^{\star *}$ & $0.077^{\star \star}$ & 0.044 & $0.054^{*}$ & $0.051^{*}$ & -0.004 & 1.000 & $0.062^{* *}$ & -0.019 & $0.058^{*}$ & 0.027 & -0.041 \\
\hline $\begin{array}{l}\text { On a sports team } \\
\text { (Intercollegiate or } \\
\text { Campus) } \\
(\text { No }=0 ; \\
\text { Yes }=1)\end{array}$ & 0.028 & -0.004 & 0.016 & -0.010 & -0.043 & $-0.082^{\star *}$ & $0.062^{\star *}$ & 1.000 & 0.007 & $0.141^{\star *}$ & -0.012 & 0.017 \\
\hline $\begin{array}{c}\text { International } \\
\text { Student } \\
(\mathrm{No}=0 ; \mathrm{Yes}=1)\end{array}$ & $0.048^{*}$ & 0.042 & 0.035 & 0.017 & $0.110^{* *}$ & 0.024 & -0.019 & 0.007 & 1.000 & 0.021 & 0.037 & $-0.317^{\star *}$ \\
\hline $\begin{array}{l}\text { Lives on } \\
\text { Campus } \\
(\text { No }=0 ; \\
\text { Yes }=1)\end{array}$ & $0.048^{*}$ & $0.067^{\star *}$ & 0.000 & $-0.055^{\star}$ & -0.044 & -0.041 & $0.058^{*}$ & $0.141^{\star *}$ & 0.021 & 1.000 & 0.036 & -0.003 \\
\hline $\begin{array}{c}\text { Live Alone } \\
(\text { No }=0 \\
\text { Yes }=1)\end{array}$ & $0.107^{\star *}$ & 0.030 & 0.029 & 0.042 & $0.066^{* *}$ & 0.030 & 0.027 & -0.012 & 0.037 & 0.036 & 1.000 & $-0.091^{\star \star}$ \\
\hline $\begin{array}{c}\text { Race } \\
(\text { Non-white }=0 ; \\
\text { White }=1)\end{array}$ & $-0.090^{\star *}$ & $-0.076^{\star *}$ & $-0.048^{*}$ & -0.032 & $-0.082^{\star *}$ & $-0.109^{\star *}$ & -0.041 & 0.017 & $-0.317^{\star \star}$ & -0.003 & $\begin{array}{c}-0.091^{*} \\
*\end{array}$ & 1.000 \\
\hline
\end{tabular}

${ }^{* *} \mathrm{p}<0.01 .{ }^{*} \mathrm{p}<0.05$. 
(b)

\begin{tabular}{|c|c|c|c|c|c|c|c|c|c|c|c|c|}
\hline 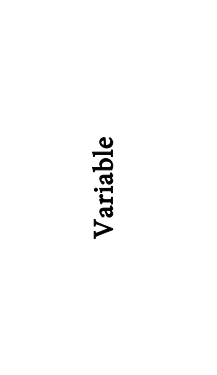 & 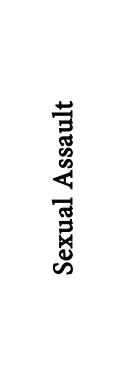 & 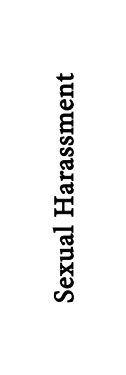 & 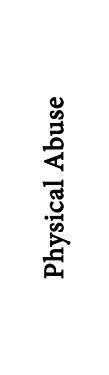 & 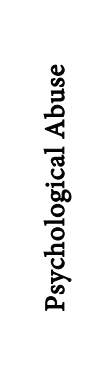 & 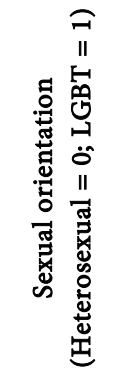 & 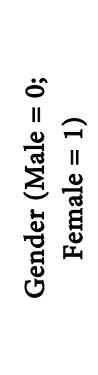 & 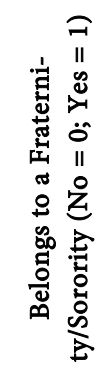 & 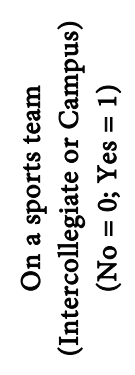 & 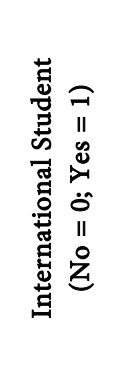 & 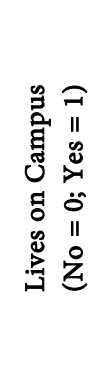 & 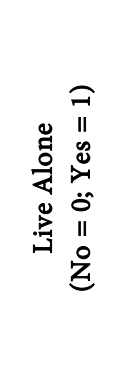 & 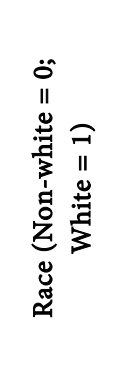 \\
\hline $\begin{array}{c}\text { Sexual } \\
\text { Assault }\end{array}$ & 1.000 & $0.258^{\star *}$ & $0.113^{* *}$ & 0.041 & $0.248^{\star *}$ & .a & $0.078^{* *}$ & 0.021 & 0.042 & 0.051 & $0.095^{* *}$ & $-0.105^{\star *}$ \\
\hline $\begin{array}{c}\text { Sexual } \\
\text { Harassment }\end{array}$ & $0.258^{\star \star}$ & 1.000 & $0.171^{\star *}$ & $0.208^{\star *}$ & $0.136^{\star *}$ & a & $0.083^{* *}$ & 0.006 & 0.047 & 0.027 & 0.049 & $-0.085^{\star *}$ \\
\hline $\begin{array}{c}\text { Physical } \\
\text { Abuse }\end{array}$ & $0.113^{\star *}$ & $0.171^{\star *}$ & 1.000 & $0.403^{* *}$ & $0.076^{*}$ & a & $0.088^{* *}$ & 0.016 & 0.024 & 0.003 & 0.003 & -0.042 \\
\hline $\begin{array}{c}\text { Psychological } \\
\text { Abuse }\end{array}$ & 0.041 & $0.208^{\star *}$ & $0.403^{* *}$ & 1.000 & 0.028 & .a & 0.053 & 0.007 & 0.022 & -0.056 & 0.001 & -0.035 \\
\hline $\begin{array}{c}\text { Sexual } \\
\text { orientation } \\
(\text { Heterosexual = } \\
0 ; \mathrm{LGBT}=1)\end{array}$ & $0.248^{\star *}$ & $0.136^{\star *}$ & $0.076^{*}$ & 0.028 & 1.000 & a & 0.058 & -0.058 & $0.127^{\star *}$ & -0.036 & 0.020 & $-0.135^{\star *}$ \\
\hline $\begin{array}{c}\text { Gender }(\text { Male }= \\
0 ; \text { Female }=1)\end{array}$ & a & .a & .a & a & a & .a & a & a & .a & a & .a & .a \\
\hline $\begin{array}{c}\text { Belongs to a } \\
\text { Fraternity/ } \\
\text { Sorority (No = } \\
0 ; \text { Yes = 1) }\end{array}$ & $0.078^{\star *}$ & $0.083^{\star *}$ & $0.088^{* *}$ & 0.053 & 0.058 & .a & 1.000 & 0.034 & -0.014 & 0.029 & 0.036 & $-0.058^{*}$ \\
\hline $\begin{array}{l}\text { On a sports } \\
\text { team } \\
\text { (Intercollegiate } \\
\text { or Campus) } \\
(\text { No }=0 ; \\
\text { Yes }=1)\end{array}$ & 0.021 & 0.006 & 0.016 & 0.007 & -0.058 & a & 0.034 & 1.000 & 0.015 & $0.097^{\star *}$ & 0.015 & 0.005 \\
\hline $\begin{array}{c}\text { International } \\
\text { Student } \\
(\text { No }=0 ; \\
\text { Yes }=1)\end{array}$ & 0.042 & 0.047 & 0.024 & 0.022 & $0.127^{\star *}$ & a & -0.014 & 0.015 & 1.000 & 0.002 & $0.109^{\star *}$ & $-0.336^{\star *}$ \\
\hline $\begin{array}{c}\text { Lives on } \\
\text { Campus (No = } \\
0 ; \text { Yes }=1)\end{array}$ & 0.051 & 0.027 & 0.003 & -0.056 & -0.036 & .a & 0.029 & $0.097^{\star *}$ & 0.002 & 1.000 & 0.030 & -0.004 \\
\hline $\begin{array}{c}\text { Live Alone } \\
(\text { No = 0; } \\
\text { Yes = 1) }\end{array}$ & $0.095^{\star *}$ & 0.049 & 0.003 & 0.001 & 0.020 & a & 0.036 & 0.015 & $0.109^{\star *}$ & 0.030 & 1.000 & $-0.126^{\star *}$ \\
\hline $\begin{array}{c}\text { Race } \\
(\text { Non-white }=0 ; \\
\text { White }=1)\end{array}$ & $-0.105^{\star *}$ & $-0.085^{\star *}$ & -0.042 & -0.035 & $-0.135^{\star *}$ & .a & $-0.058^{*}$ & 0.005 & $-0.336^{* *}$ & -0.004 & $-0.126^{\star *}$ & 1.000 \\
\hline
\end{tabular}

${ }^{* *} \mathrm{p}<0.01 .{ }^{*} \mathrm{p}<0.05$. 
(c)

\begin{tabular}{|c|c|c|c|c|c|c|c|c|c|c|c|c|}
\hline 苞 & 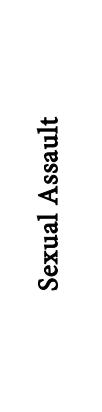 & 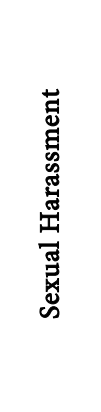 & 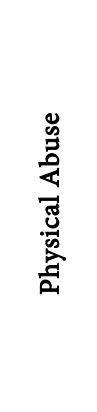 & 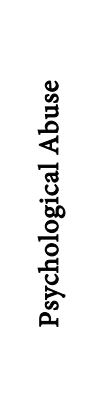 & 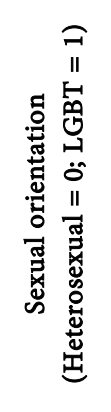 & 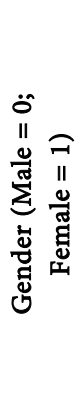 & 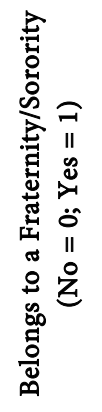 & 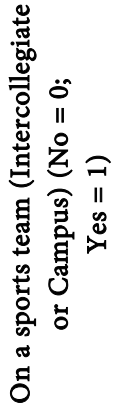 & 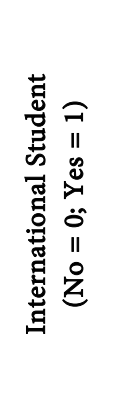 & 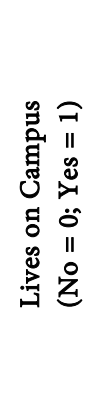 & 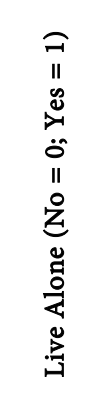 & 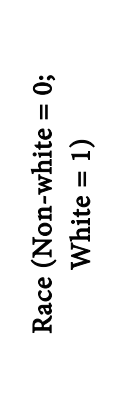 \\
\hline Sexual Assault & 1.000 & $0.164^{\star *}$ & $0.228^{\star *}$ & $0.191^{\star *}$ & $0.113^{\star *}$ & .a & 0.054 & 0.069 & 0.048 & 0.061 & $0.114^{* *}$ & -0.047 \\
\hline Sexual Harassment & $0.164^{* *}$ & 1.000 & 0.044 & $0.211^{\star *}$ & 0.015 & a & $0.083^{\star}$ & 0.067 & 0.020 & $0.183^{\star *}$ & -0.022 & 0.021 \\
\hline Physical Abuse & $0.228^{\star *}$ & 0.044 & 1.000 & $0.358^{\star *}$ & $0.087^{\star}$ & .a & -0.026 & 0.015 & 0.052 & -0.006 & 0.069 & -0.060 \\
\hline $\begin{array}{c}\text { Psychological } \\
\text { Abuse }\end{array}$ & $0.191^{\star *}$ & $0.211^{\star *}$ & $0.358^{* *}$ & 1.000 & $0.113^{* *}$ & a & 0.056 & -0.027 & 0.006 & -0.046 & $0.094^{*}$ & -0.012 \\
\hline $\begin{array}{l}\text { Sexual orientation } \\
\text { (Heterosexual }=0 ; \\
\text { LGBT }=1)\end{array}$ & $0.113^{* *}$ & 0.015 & $0.087^{\star}$ & $0.113^{\star *}$ & 1.000 & a & 0.047 & -0.009 & $0.091^{\star}$ & -0.047 & $0.108^{\star *}$ & -0.011 \\
\hline $\begin{array}{c}\text { Gender }(\text { Male }=0 ; \\
\text { Female }=1)\end{array}$ & a & a & a & a & a & a & a & a & a & a & a & a \\
\hline $\begin{array}{l}\text { Belongs to a } \\
\text { Fraternity/Sorority } \\
(\mathrm{No}=0 ; \text { Yes }=1)\end{array}$ & 0.054 & $0.083^{\star}$ & -0.026 & 0.056 & 0.047 & a & 1.000 & $0.113^{\star *}$ & -0.026 & $0.103^{* *}$ & 0.014 & -0.020 \\
\hline $\begin{array}{l}\text { On a sports team } \\
\text { (Intercollegiate or } \\
\text { Campus) }(\text { No }=0 ; \\
\text { Yes }=1)\end{array}$ & 0.069 & 0.067 & 0.015 & -0.027 & -0.009 & a & $0.113^{* *}$ & 1.000 & -0.001 & $0.214^{\star *}$ & -0.050 & 0.014 \\
\hline $\begin{array}{c}\text { International } \\
\text { Student }(\text { No }=0 ; \\
\text { Yes }=1)\end{array}$ & 0.048 & 0.020 & 0.052 & 0.006 & $0.091^{\star}$ & a & -0.026 & -0.001 & 1.000 & 0.050 & -0.059 & $-0.292^{\star *}$ \\
\hline $\begin{array}{l}\text { Lives on Campus } \\
(\text { No }=0 ; \text { Yes }=1)\end{array}$ & 0.061 & $0.183^{\star *}$ & -0.006 & -0.046 & -0.047 & .a & $0.103^{\star *}$ & $0.214^{\star *}$ & 0.050 & 1.000 & 0.048 & -0.012 \\
\hline $\begin{array}{c}\text { Live Alone } \\
(\text { No }=0 ; \text { Yes }=1)\end{array}$ & $0.114^{\star *}$ & -0.022 & 0.069 & $0.094^{*}$ & $0.108^{\star *}$ & a & 0.014 & -0.050 & -0.059 & 0.048 & 1.000 & -0.041 \\
\hline $\begin{array}{l}\text { Race (Non-white } \\
=0 ; \text { White }=1 \text { ) }\end{array}$ & -0.047 & 0.021 & -0.060 & -0.012 & -0.011 & a & -0.020 & 0.014 & $-0.292^{\star *}$ & -0.012 & -0.041 & 1.000 \\
\hline
\end{tabular}

${ }^{* *} \mathrm{p}<0.01 .{ }^{*} \mathrm{p}<0.05$.

genders (column 1 in Table 4(a)), males, and females as well. The effect was quite strong in that one was almost four times $(\operatorname{Exp}(B)=3.907)$ more likely to report sexual assault if they were a sexual minority. Sexual minority males were almost ten times $(\operatorname{Exp}(B)=9.449)$ more likely to report sexual assault victimization than heterosexual males. Sexual minority females were a little over two times as likely to report sexual assault as heterosexual females $(\operatorname{Exp}(B)=2.159)$. These findings indicate that the effect of sexual orientation on sexual assault victimization was much stronger for males than for females. 
Table 4. (a) Victimization outcomes for sexual assault. (b) Victimization outcomes for sexual harassment. (c) Victimization outcomes for physical Abuse. (d) Victimization outcomes for psychological Abuse.

(a)

\begin{tabular}{|c|c|c|c|c|c|c|c|c|c|}
\hline \multirow{3}{*}{$\begin{array}{c}\text { Independent Variable } \\
\text { Sexual Orientation } \\
\text { (1 = Gay/Lesbian/Other })\end{array}$} & \multicolumn{3}{|c|}{ All Cases } & \multicolumn{3}{|c|}{ Male } & \multicolumn{3}{|c|}{ Female } \\
\hline & \multirow{2}{*}{$\begin{array}{c}\text { B } \\
1.363\end{array}$} & \multicolumn{2}{|c|}{$\operatorname{Exp}(B)$} & \multirow{2}{*}{$\begin{array}{c}\text { B } \\
2.246\end{array}$} & \multicolumn{2}{|c|}{$\operatorname{Exp}(B)$} & \multirow{2}{*}{$\begin{array}{c}\text { B } \\
0.770\end{array}$} & \multicolumn{2}{|c|}{$\operatorname{Exp}(B)$} \\
\hline & & 3.907 & ** & & 9.449 & $* *$ & & 2.159 & $* *$ \\
\hline Gender ( 1 = female $)$ & 0.882 & 2.416 & ** & & & & & & \\
\hline Fraternity/Sorority & 0.398 & 1.489 & + & 0.493 & 1.637 & & 0.284 & 1.328 & \\
\hline $\begin{array}{c}\text { On a Sports Team } \\
\text { (Intercollegiate or Campus) }\end{array}$ & 0.327 & 1.387 & & 0.290 & 1.337 & & 0.456 & 1.578 & \\
\hline International Student & 0.116 & 1.123 & & -0.241 & 0.786 & & 0.348 & 1.416 & \\
\hline Lives on Campus & 0.346 & 1.413 & * & 0.441 & 1.555 & + & 0.247 & 1.280 & \\
\hline Live Alone & 0.729 & 2.074 & ** & 0.821 & 2.272 & * & 0.757 & 2.133 & $* *$ \\
\hline Race $(1=$ White $)$ & -0.384 & 0.681 & * & -0.529 & 0.589 & + & -0.191 & 0.826 & \\
\hline Chi-Square & & 177.079 & & & 26.238 & & & 38.210 & \\
\hline $\mathrm{DF}$ & & 8 & & & 7 & & & 7 & \\
\hline $\mathbf{N}$ & & 1881 & & & 1143 & & & 738 & \\
\hline
\end{tabular}

${ }^{* *} \mathrm{p}<0.01 .{ }^{*} \mathrm{p}<0.05 .+\mathrm{p}<0.10$.

(b)

\begin{tabular}{|c|c|c|c|c|c|c|c|c|c|}
\hline \multirow{3}{*}{$\begin{array}{c}\text { Independent Variable } \\
\text { Sexual Orientation } \\
(1 \text { = Gay/Lesbian/Other) }\end{array}$} & \multicolumn{3}{|c|}{ All Cases } & \multicolumn{3}{|c|}{ Male } & \multicolumn{3}{|c|}{ Female } \\
\hline & \multirow{2}{*}{$\begin{array}{c}\text { B } \\
0.717\end{array}$} & \multicolumn{2}{|c|}{$\operatorname{Exp}(B)$} & \multirow{2}{*}{$\begin{array}{c}\text { B } \\
1.249\end{array}$} & \multicolumn{2}{|c|}{$\operatorname{Exp}(B)$} & \multirow{2}{*}{$\begin{array}{c}\text { B } \\
0.193\end{array}$} & \multicolumn{2}{|c|}{$\operatorname{Exp}(B)$} \\
\hline & & 2.049 & $* *$ & & 3.489 & $* *$ & & 1.212 & \\
\hline Gender $(1=$ female $)$ & 1.500 & 4.483 & ** & & & & & & \\
\hline Fraternity/Sorority & 0.495 & 1.640 & ** & 0.449 & 1.566 & * & 0.523 & 1.686 & + \\
\hline $\begin{array}{c}\text { On a Sports Team } \\
\text { (Intercollegiate or Campus) }\end{array}$ & 0.084 & 1.088 & & 0.040 & 1.040 & & 0.159 & 1.172 & \\
\hline International Student & 0.180 & 1.197 & & 0.083 & 1.087 & & 0.165 & 1.179 & \\
\hline Lives on Campus & 0.362 & 1.436 & $* *$ & 0.119 & 1.126 & & 0.782 & 2.186 & $* *$ \\
\hline Live Alone & 0.057 & 1.059 & & 0.248 & 1.281 & & -0.216 & 0.806 & \\
\hline Race $(1=$ White $)$ & -0.123 & 0.885 & & -0.287 & 0.750 & + & 0.140 & 1.150 & \\
\hline Chi-Square & 233 & 35.325 & & & 53.655 & & & 58.078 & \\
\hline DF & & 8 & & & 7 & & & 7 & \\
\hline $\mathbf{N}$ & & 881 & & & 143 & & & 738 & \\
\hline
\end{tabular}

${ }^{* *} \mathrm{p}<0.01 .{ }^{*} \mathrm{p}<0.05 .+\mathrm{p}<0.10$. 
(c)

\begin{tabular}{|c|c|c|c|c|c|c|c|c|c|}
\hline \multirow{3}{*}{$\begin{array}{c}\text { Independent Variable } \\
\text { Sexual Orientation } \\
\text { (1 = Gay/Lesbian/Other) }\end{array}$} & \multicolumn{3}{|c|}{ All Cases } & \multicolumn{3}{|c|}{ Male } & \multicolumn{3}{|c|}{ Female } \\
\hline & \multirow{2}{*}{$\begin{array}{c}\text { B } \\
0.634\end{array}$} & \multicolumn{2}{|c|}{$\operatorname{Exp}(B)$} & \multirow{2}{*}{$\begin{array}{c}\text { B } \\
0.684\end{array}$} & \multicolumn{2}{|c|}{$\operatorname{Exp}(B)$} & \multirow{2}{*}{$\begin{array}{c}\text { B } \\
0.578\end{array}$} & \multicolumn{2}{|c|}{$\operatorname{Exp}(B)$} \\
\hline & & 1.885 & $* *$ & & 1.981 & * & & 1.782 & * \\
\hline Gender $(1=$ female $)$ & -0.103 & 0.902 & & & & & & & \\
\hline Fraternity/Sorority & 0.262 & 1.300 & & 0.547 & 1.728 & ** & -0.261 & 0.770 & \\
\hline $\begin{array}{l}\text { On a Sports Team } \\
\text { (Intercollegiate } \\
\text { or Campus) }\end{array}$ & 0.105 & 1.111 & & 0.102 & 1.107 & & 0.188 & 1.206 & \\
\hline International Student & 0.153 & 1.165 & & 0.084 & 1.087 & & 0.307 & 1.359 & \\
\hline Lives on Campus & -0.016 & 0.984 & & 0.007 & 1.007 & & -0.045 & 0.956 & \\
\hline Live Alone & 0.156 & 1.169 & & -0.052 & 0.949 & & 0.442 & 1.556 & + \\
\hline Race $(1=$ White $)$ & -0.193 & 0.824 & & -0.145 & 0.865 & & -0.248 & 0.780 & \\
\hline Chi-Square & & 6.453 & & & 01.398 & & & 57.276 & \\
\hline DF & & 8 & & & 7 & & & 7 & \\
\hline $\mathrm{N}$ & & 881 & & & 1143 & & & 738 & \\
\hline
\end{tabular}

${ }^{* *} \mathrm{p}<0.01 .{ }^{*} \mathrm{p}<0.05 .+\mathrm{p}<0.10$.

(d)

\begin{tabular}{|c|c|c|c|c|c|c|c|c|c|}
\hline \multirow{3}{*}{$\begin{array}{c}\text { Independent Variable } \\
\text { Sexual Orientation } \\
(1=\text { Gay/Lesbian/Other })\end{array}$} & \multicolumn{3}{|c|}{ All Cases } & \multicolumn{3}{|c|}{ Male } & \multicolumn{3}{|c|}{ Female } \\
\hline & \multirow{2}{*}{$\begin{array}{c}\text { B } \\
0.499\end{array}$} & \multicolumn{2}{|c|}{$\operatorname{Exp}(B)$} & \multirow{2}{*}{$\begin{array}{c}\text { B } \\
0.192\end{array}$} & \multicolumn{2}{|c|}{$\operatorname{Exp}(B)$} & \multirow{2}{*}{$\begin{array}{c}\text { B } \\
0.697\end{array}$} & \multicolumn{2}{|c|}{$\operatorname{Exp}(B)$} \\
\hline & & 1.647 & * & & 1.212 & & & 2.008 & ** \\
\hline Gender $(1=$ female $)$ & 0.221 & 1.248 & * & & & & & & \\
\hline Fraternity/Sorority & 0.331 & 1.392 & * & 0.324 & 1.382 & + & 0.365 & 1.441 & \\
\hline $\begin{array}{c}\text { On a Sports Team } \\
\text { (Intercollegiate or } \\
\text { Campus) }\end{array}$ & 0.009 & 1.009 & & 0.064 & 1.067 & & -0.114 & 0.892 & \\
\hline International Student & 0.047 & 1.048 & & 0.106 & 1.112 & & 0.039 & 1.039 & \\
\hline Lives on Campus & -0.228 & 0.796 & * & -0.245 & 0.783 & * & -0.199 & 0.820 & \\
\hline Live Alone & 0.235 & 1.265 & & -0.033 & 0.967 & & 0.541 & 1.717 & * \\
\hline Race $(1=$ White $)$ & -0.067 & 0.936 & & -0.132 & 0.876 & & -0.020 & 0.980 & \\
\hline Chi-Square & & 497.506 & & & 499.703 & & & 992.033 & \\
\hline DF & & 8 & & & 7 & & & 7 & \\
\hline $\mathrm{N}$ & & 1881 & & & 1143 & & & 738 & \\
\hline
\end{tabular}

${ }^{* *} \mathrm{p}<0.01 .{ }^{*} \mathrm{p}<0.05 .+\mathrm{p}<0.10$.

Both non-sexual minority and sexual minority females were over two times more likely to report sexual assault victimization than were males of any sexual orientation $(\operatorname{Exp}(B)=2.416)$. Living on campus increased the reporting of victimization for the full sample $(\operatorname{Exp}(B)=1.413)$ and for males $(\operatorname{Exp}(B)=1.555)$, but not for females. Whites were less likely to report sexual assault $(\operatorname{Exp}(\mathrm{B})=$ 0.681) than non-Whites. For males, Whites were less likely to report sexual assault $(\operatorname{Exp}(B)=0.589)$, but there was no difference by race for females. Living 
alone had increased the likelihood of sexual assault for the full sample $(\operatorname{Exp}(B)=$ $2.074)$, males $(\operatorname{Exp}(B)=2.272)$, and females $(\operatorname{Exp}(B)=2.133)$. Fraternity/sorority membership increased reported sexual assault victimization in the full sample $(\operatorname{Exp}(B)=1.489)$, but did not impact reported victimization in either the male or female samples.

Sexual minority students were about two times $(\operatorname{Exp}(B)=2.049)$ more likely to report sexual harassment (see column 1 in Table 4(b)) than non-sexual minority students. Sexual minority males were over three times $(\operatorname{Exp}(B)=3.489)$ more likely to report sexual harassment than heterosexual males. Sexual minority females did not report more sexual harassment than non-sexual minority females. Gender did have an impact on the reporting of sexual harassment. Women were over four times $(\operatorname{Exp}(B)=4.483)$ more likely to have reported sexual harassment than were men. The findings that females were significantly more likely to report sexual harassment than males and that sexual minority females were not more likely to report sexual harassment than non-sexual minority females suggests that the driving force for sexual harassment for females is simply their gender.

Sexual harassment was more likely to be reported by those in a fraternity or sorority. This finding holds for all three samples: all cases $(\operatorname{Exp}(B)=1.640)$, males $(\operatorname{Exp}(B)=1.566)$, and females $(\operatorname{Exp}(B)=1.686)$. Living on campus increased the likelihood of reported sexual harassment for the full sample $(\operatorname{Exp}(B)=1.436)$ and for females $(\operatorname{Exp}(B)=2.186)$, but not for males. White males were less likely to report sexual harassment $(\operatorname{Exp}(B)=0.750)$ than non-Whites, but race had no effect in the full sample or for females. The other control variables did not exhibit significant relationships with reported sexual harassment.

Table 4(c) provides the results for physical abuse. Similar to sexual assault, sexual minority students were significantly more likely to have reported physical abuse than non-sexual minority students. However, the impact of sexual minority status was somewhat similar across the samples (all cases, males, and females) for physical abuse. Sexual minority males were about two times $(\operatorname{Exp}(B)=1.981)$ more likely to report physical abuse than non-sexual minority males. The full sample found that sexual minority students were also just under two times $(\operatorname{Exp}(B)=1.885)$ more likely to report physical abuse victimization. For females, sexual minority students were less than two times $(\operatorname{Exp}(B)=1.782)$ more likely to report victimization than non-sexual minority students.

Unlike sexual assault and harassment, gender had no impact on the reporting of physical abuse. Membership in a fraternity or sorority increased the likelihood of reporting physical victimization, for males $(\operatorname{Exp}(B)=1.728)$, but not for the full sample or females. Living alone increased the reporting of physical abuse for females $(\operatorname{Exp}(\mathrm{B})=1.556)$. None of the other variables had significant relationships with reported physical abuse.

For the final type of victimization, psychological abuse, sexual minority students were significantly more likely to report victimization than were heterosexual students. Unlike the results for both sexual assault and physical abuse, 
this finding holds for the full sample $(\operatorname{Exp}(\mathrm{B})=1.647)$ and females $(\operatorname{Exp}(\mathrm{B})=$ 2.008), but not for males. Thus sexual minority males were not more likely to report psychological abuse than non-minority sexual males.

Females were more likely to report psychological abuse than males $(\operatorname{Exp}(B)=$ 1.248). Membership in a fraternity or sorority increased reported psychological abuse in the full $(\operatorname{Exp}(B)=1.392)$ and male samples $(\operatorname{Exp}(B)=1.382)$. Living on campus reduced the likelihood of reported psychological abuse for the full sample $(\operatorname{Exp}(B)=0.796)$ and the male sample $(\operatorname{Exp}(B)=0.783)$. Living alone increased the likelihood of reported psychological abuse for females only $(\operatorname{Exp}(B)=$ 1.717).

\section{Discussion}

While research on violence among college students has historically neglected the victimization experiences of sexual minority college students, the findings clearly point to an effect of sexual orientation on reported victimization. Consistent with previous research (Edwards et al., 2015) sexual minority students were significantly more likely to report at least one incident of sexual assault $(\operatorname{Exp}(B)=$ 3.907), sexual harassment $(\operatorname{Exp}(B)=2.049)$, physical abuse $(\operatorname{Exp}(B)=1.885)$, and psychological abuse $(\operatorname{Exp}(B)=1.647)$ than non-sexual minority students . These findings lend support to the hypothesis that sexual minority students would be more likely to report victimization experiences in all of the categories. If we assume that sexual minority students were in same sex relationships, these findings support the hypothesis that same sex couples will report the highest rates of psychological abuse, with non-sexual minority females reporting more incidents than males. Findings indicate that males, however, have a greater risk of other types of abuse.

Sexual minority males were more likely to report each of the types of abuse (sexual assault $(\operatorname{Exp}(B)=9.449)$, sexual harassment $(\operatorname{Exp}(B)=3.489)$, and physical abuse $(\operatorname{Exp}(B)=1.981))$, except for psychological abuse. In fact, sexual minority males' odds of being victimized were consistently larger than they were for the full sample. This suggests that except for psychological abuse, sexual minority status for males is a bigger predictor of victimization than it is for females (though it appears to matter for both genders). This supports the hypothesis that sexual minority males would experience higher rates of sexual victimization compared to non-sexual minority males. For females, sexual minority status increases the likelihood of victimization for three of the four types of abuse (sexual assault $(\operatorname{Exp}(B)=2.159)$, physical abuse $(\operatorname{Exp}(B)=1.782)$, and psychological abuse $(\operatorname{Exp}(B)=2.008))$. Sexual minority females did not see an increased risk of victimization for sexual harassment. Females were significantly more likely to report sexual harassment, thus this suggests that being female is the driving force for why females are sexually harassed. These findings lend partial support for our hypothesis that women would experience higher victimization rates in all abuse categories regardless of sexual orientation. The findings have important program 
development implications directed at reducing victimization via education.

The current study is unique because the findings highlight the importance for education among groups about their varying risks for different types of abuse. For example, since psychological abuse is more common for sexual minority female students, they may be exposed to unique forms of abuse such as the threat of "outing" a partner (i.e., revealing sexual orientation) to others (Carvalho, Lewis, Derlega, A., \& Viggiano, 2011). These are relevant indicators for policy development, not only for students but for other employees at colleges and universities.

This study is also important because it speaks to the need for colleges to have adequate policies for faculty and staff as well. While it varied depending on academic program, previous research found that student affairs staff members were more aware of issues than were faculty members, with findings indicating a greater knowledge of sexual minority topics and events amongst the student affairs staff when compared to the faculty (R. D. Brown et al., 2004). Clear policies that faculty and staff are aware of can assist them in recognizing and addressing these issues whenever observed, as well as being receptive to students who bring up such issues with them. It is important that faculty and staff should be kept aware of gender differences in experiences of abuse. Gender was also significantly related to reported sexual assault, sexual harassment, and psychological abuse, but not physical abuse. Thus, gender matters for three of the four types of victimization and females consistently reported more victimization for all three.

The lack of a significant result between gender and physical abuse is not surprising. Prior research has not found that the likelihood of reporting physical abuse is affected by the victim's gender (Hines \& Saudino, 2003). Straus \& Ramirez (2007) report that women were significantly more likely to be seriously injured than men in cases of intimate partner physical abuse. We only examined the likelihood of physical abuse and not the extent of such abuse. Future research should examine the relationship between gender and the severity of physical abuse victimization.

Another possible reason for the lack of a relationship between gender and physical violence could be the skewed gender ratio at RIT. RIT has many more male students than female students. Despite the fact that our sample is about $40 \%$ female, over two-thirds of the students at RIT are male. Guttentag and Secord (1983) argue that when women are scarce and men are plentiful, men will be less likely to harass or assault women. Female college students at RIT may be less likely to be physically victimized by intimate partners because the sex ratio leads men to be more protective of women. Another possibility could be that female college students at RIT have more options to choose from amongst the male population and thus can be more selective and are better able to avoid abusive relationships.

While the current analysis uses sexual harassment as a variable, future research may wish to look at sexual harassment alongside measures of general harassment, 
such as bullying. This was an area of victimization the current study could not examine. There were no questions concerning overall bullying or harassment by others. There were measures of intimate partner abuse, both physical and psychological, and sexual assault, but sexual minority victimization was more common for both IPV and for sexual assaults that are not necessarily perpetrated by an intimate partner. Thus, an examination of overall harassment of sexual minority students by non-intimate partners might be helpful to have alongside the victimization categories used here.

Our research indicates that sexual orientation is a strong predictor of victimization, particularly for males. The results of this study illustrate the importance for college health professionals and others dealing with college populations of avoiding a "one size fits all" policy approach to addressing partner violence, sexual harassment and sexual abuse. Accordingly, when developing programs and services, practitioners and college health professionals must strive for inclusivity, as well as develop targeted approaches for outreach to populations on their campuses, which may be at greater risk.

Although the current study extends research on IPV, sexual assault, and sexual harassment among sexual minority college students, findings should be viewed with caution in light of several limitations. First, data were obtained by self-report. Thus, the possibility of deliberate response distortion must be considered. Second, present findings may not generalize beyond the particular sample. We note our sample consisted of a small number of college men and women that were sexual minority on a mid-size campus who may differ from other groups in their experiences of perceived psychological, physical, and sexual abuse, and sexual harassment. The study does, however, provide evidence for future comparisons.

\section{Fund}

This project was supported by funding awarded by the Office on Violence Against Women, Office of Justice Programs, U.S. Department of Justice. Points of view in this document are those of the authors and do not necessarily represent the official position or policies of the U.S. Department of Justice.

\section{References}

Anderson, M. L., \& Leigh, I. W. (2010). Internal Consistency and Factor Structure of the Revised Conflict Tactics Scales in a Sample of Deaf Female College Students. Journal of Family Violence, 25, 475-483. https://doi.org/10.1007/s10896-010-9308-6

Avant, E. M., Swopes, R. M., Davis, J. L., \& Elhai, J. D. (2011). Psychological Abuse and Posttraumatic Stress Symptoms in College Students. Journal of Interpersonal Violence, 26, 3080-3097. https://doi.org/10.1177/0886260510390954

Banyard, V. L., Ward, S. K., Cohn, E. S., Plante, E. G., Moorhead, C., \& Walsh, W. (2007). Unwanted Sexual Contact on Campus: A Comparison of Women's and Men's Experiences. Violence and Victims, 22, 52-70.

https://doi.org/10.1891/vv-v22i1a004 
Bartholomew, K., Regan, K. V., Oram, D., \& White, M. A. (2008). Correlates of Partner Abuse in Male Same-Sex Relationships. Violence and Victims, 23, 344-360. https://doi.org/10.1891/0886-6708.23.3.344

Boeringer, S. B. (1996). Influences of Fraternity Membership, Athletics, and Male Living Arrangements on Sexual Aggression. Violence against Women, 2, 134-147. https://doi.org/10.1177/1077801296002002002

Brown, M. J., \& Groscup, J. (2009). Perceptions of Same-Sex Domestic Violence among Crisis Center Staff. Journal of Family Violence, 24, 87-93. https://doi.org/10.1007/s10896-008-9212-5

Brown, R. D., Clarke, B., Gortmaker, V., \& Robinson-Keilig, R. (2004). Assessing the Campus Climate for Gay, Lesbian, Bisexual and Transgender (GLBT) Students using a Multiple Perspectives Approach. Journal of College Student Development, 45, 8-26. https://doi.org/10.1353/csd.2004.0003

Burke, L. K., \& Follingstad, D. R. (1999). Violence in Lesbian and Gay Relationships: Theory, Prevalence, and Correlational Factors. Clinical Psychology Review, 19, 487-512.

Caetano, R., Schafer, J., \& Cunradi, C. B. (2001). Alcohol-Related Intimate Partner Violence among White, Black and Hispanic Couples in the United States. Alcohol Research and Health, 28, 58-65.

Carvalho, A. F., Lewis, R. J., Derlega, V. J., A., W. B., \& Viggiano, C. (2011). Internalized Sexual Minority Stressors and Same-Sex Intimate Partner Violence. Journal of Family Violence, 26, 501-509. https://doi.org/10.1007/s10896-011-9384-2

Cercone, J. J., Beach, S. R. H., \& Arias, I. (2005). Gender Symmetry in Dating Intimate Partner Violence Does Similar Behavior Imply Similar Constructs? Violence and Victims, 20, 207-218. https://doi.org/10.1891/vivi.2005.20.2.207

Coston, C. T. M. (2004). Worries about Crime among Foreign Students Studying in the United States: A Comparative Study. In C. T. M. Coston (Ed.), Victimizing Vulnerable Groups: Irimages of Uniquely High-Risk Crime Targets (pp. 173-193). Westport, CT: Praeger.

Cotten-Huston, A. L., \& Waite, B. M. (2000). Anti-Homosexual Attitudes in College Students: Predictors and Classroom Interventions. Journal of Homosexuality, 38, 117-133. https://doi.org/10.1300/J082v38n03_07

D’Augelli, A. R. (1992). Lesbians and Gay Male Undergraduates' Experiences of Harassment and Fear on Campus. Journal of Interpersonal Violence, 7, 383-395. https://doi.org/10.1177/088626092007003007

Duncan, D. F. (1990). Prevalence of Sexual Assault Victimization among Heterosexual and Gay/Lesbian University Students. Psychological Reports, 66, 65-66.

https://doi.org/10.2466/pr0.1990.66.1.65

Edwards, K. M., Sylaska, K. M., Barry, J. E., Moynihan, M. M., Banyard, V. L., Cohn, E. S., Ward, S. K. et al. (2015). Physical Dating Violence, Sexual Violence, and Unwanted Pursuit Victimization: A Comparison of Incidence Rates among Sexual-Minority and Heterosexual College Students. Journal of Interpersonal Violence, 30, 580-600. https://doi.org/10.1177/0886260514535260

Fasting, K., Brackenridge, C. H., \& Sungot-Borgen, J. (2003). Experiences of Sexual Harassment and Abuse amongst Norwegian Elite Female Athletes and Non-Athletes. Research Quarterly for Exercise and Sport, 74, 84-97. https://doi.org/10.1080/02701367.2003.10609067

Fisher, B. S., Cullen, F. T., \& Turner, M. G. (2000). The Sexual Victimization of College Women. Washington DC: National Institute of Justice. 
Follingstad, D. R., \& DeHart, D. D. (2000). Defining Psychological Abuse of Husbands toward Wives: Contexts, Behaviors and Typologies. Journal of Interpersonal Violence, 15, 891-920. https://doi.org/10.1177/088626000015009001

Franklin, C. A., Bouffard, L. A., \& Pratt, T. C. (2012). Sexual Assault on the College Campus: Fraternity Affiliation, Male Peer Support, and Low Self-Control. Criminal Justice and Behavior, 39, 1457-1480. https://doi.org/10.1177/0093854812456527

Guttentag, M., \& Secord, P. F. (1983). Too Many Women? The Sex Ratio Question. Beverly Hills, CA: Sage.

Harned, M. S. (2001). Abused Women or Abused Men? An Examination of the Context and Outcomes of Dating Violence. Violence and Victims, 16, 269-285.

Hines, D. A., \& Saudino, K. J. (2003). Gender Differences Is Psychological, Physical, and Sexual Aggression among College Students using the Revised Conflicts Tactics Scales. Violence and Victims, 18, 197-217. https://doi.org/10.1891/vivi.2003.18.2.197

Koss, M. P., Gidycz, C. A., \& Wisniewski, N. (1987). The Scope of Rape: Incidence and Prevalence of Sexual Aggression and Victimization in a National Sample of Higher Education Students. Journal of Consulting and Clinical Psychology, 55, 162-170. https://doi.org/10.1037/0022-006X.55.2.162

Lane, K. E., \& Gwartney-Gibbs, P. A. (1985). Violence in the Context of Dating and Sex. Journal of Family Issues, 6, 45-59. https://doi.org/10.1177/019251385006001004

Lehrer, J. A., Lehrer, V. L., Lehrer, E. L., \& Oyarzun, P. B. (2007). Prevalence of and Risk Factors for Sexual Victimization in College Women in Chile. International Family Planning, 33, 168-175. https://doi.org/10.1363/3316807

Lockhart, L. L., White, B. W., Causby, V., \& Issac, A. (1994). Letting out the Secret: Violence in Lesbian Relationships. Journal of Interpersonal Violence, 9, 469-492. https://doi.org/10.1177/088626094009004003

Makepeace, J. M. (1987). Social Factors and Victim Offender Differences in Courtship Violence. Family Relations, 36, 87-91. https://doi.org/10.2307/584654

Martin, P. Y., \& Hummer, R. A. (1989). Fraternities and Rape on Campus. Gender and Society, 3, 457-473. https://doi.org/10.1177/089124389003004004

Monks, S. M., Tomaka, J., Palacios, R., \& Thompson, S. E. (2010). Sexual Victimization in Female and Male College Students: Examining the Roles of Alcohol Use, Alcohol Expectancies, and Sexual Sensation Seeking. Substance Use \& Misuse, 45, 2258-2280. https://doi.org/10.3109/10826081003694854

Orcutt, H. K., Garcia, M., \& Pickett, S. M. (2005). Women-Perpetrated Intimate Partner Violence and Romantic Attachment Style in a College Student Sample. Violence and Victims, 20, 287-302. https://doi.org/10.1891/vivi.20.3.287

Perry, A. R., \& Fromuth, M. E. (2005). Courtship Violence using Couple Data: Charactertistics and Perceptions. Journal of Interpersonal Violence, 20, 1078-1095. https://doi.org/10.1177/0886260505278106

Rankin, S. R. (2003). Campus Climate for Gay, Lesbian, Bisexual and Transgender People: A National Perspective. The Policy Institute of the National Gay and Lesbian Task Force.

Rothman, E. F., Exner, D., \& Baughman, A. L. (2011). The Prevalence of Sexual Assault against People Who Identify as Gay, Lesbian, or Bisexual in the United States: A Systematic Review. Trauma, Violence and Abuse, 12, 55-66. https://doi.org/10.1177/1524838010390707

Rouse, L. P. (1988). Abuse in Dating Relationships: A Comparison of Blacks, Whites and Hispanics. Journal of College Student Development, 29, 312-319. 
Smith, P. H., White, J. W., \& Holland, L. J. (2003). A Longitudinal Perspective on Dating Violence among Adolescent and College-Age Women. American Journal of Public Health, 93, 1104-1109. https://doi.org/10.2105/AJPH.93.7.1104

SPSS Inc. Released (2008). SPSS Statistics for Windows, Version 17.0. Chicago: SPSS Inc.

Straus, M. A., Hamby, S. L., Boney-McCoy, S., \& Sugarman, D. B. (1996). The Revised Conflict Tactics Scale (CTS2): Development and Preliminary Psychometric Data. Journal of Family Issues, 17, 283-316. https://doi.org/10.1177/019251396017003001

Todahl, J. L., Linville, D., Amy, Wheeler, J., \& Gau, J. (2009). Sexual Assault Support Services and Community Systems: Understanding Critical Issues and Needs in the LGBTQ Community. Violence against Women, 15, 952-976. https://doi.org/10.1177/1077801209335494

U. S. Department of Justice (2000). Bureau of Justice Statistics Special Report: Intimate Partner Violence. http://bjs.ojp.usdoj.gov/index.cfm?ty=pbdetail\&;iid=1002

Waldner-Haugrud, L. K., \& Vaden Gratch, L. (1997). Sexual Coercion in Gay/Lesbian Relationships: Descriptives and Gender Differences. Violence and Victims, 21, 87-98.

Wilson, H. W., \& Spatz Widom, C. (2010). Does Physical Abuse, Sexual Abuse, or Neglect in Childhood Increase the Likelihood of Same-Sex Sexual Relationships and Cohabitation? A Prospective 30-Year Follow-Up. Archives of Sexual Behavior, 39, 63-74. https://doi.org/10.1007/s10508-008-9449-3

World Health Organization (2012). Global Campaign for Violence Prevention. Violence Prevention Alliance.

http://www.who.int/violenceprevention/approach/definition/en/index.html

\section{Submit or recommend next manuscript to SCIRP and we will provide best} service for you:

Accepting pre-submission inquiries through Email, Facebook, LinkedIn, Twitter, etc. A wide selection of journals (inclusive of 9 subjects, more than 200 journals)

Providing 24-hour high-quality service

User-friendly online submission system

Fair and swift peer-review system

Efficient typesetting and proofreading procedure

Display of the result of downloads and visits, as well as the number of cited articles

Maximum dissemination of your research work

Submit your manuscript at: http://papersubmission.scirp.org/

Or contact psych@scirp.org 